\title{
Therapeutical Approach of Osteoporosis a Multidisciplinary Issue
}

\author{
Gliga Camelia¹, Marcu Simona¹, Gliga M² \\ ${ }^{1}$ Department of Histology, University of Medicine and Pharmacy, Tîrgu Mureș, Romania \\ 2 Obstetrics and Ginecology Clinic No. 2, University of Medicine and Pharmacy, Tîrgu Mureș, Romania
}

Osteoporosis is the most frequent systemic disease of the bone, that affects elderly, mainly women in menopause. It can be defined by lowering of bone mass and microarchitectural deterioration of the bone tissue, resulting in an increased bone fragility. Main complications of osteoporosis are fractures of the vertebrae, hips and forearm. In view of its large variety of causes and manifestations, diagnostic and therapeutical approach in osteoporosis represents a multidisciplinary issue. The accurate diagnosis of osteoporosis is based on a method that measures the bone mineral density, expressed by the T-score, using dual energy X-ray absorptiometry, so called DXA. Lately, in practice in order for establishing the risk of osteoporosis and osteoporotic fracture the FRAX tool is increasingly used (The Fracture Risk Assessment). Treatment of osteoporosis is complex involving non-pharmacological and pharmacological measures. Non-pharmacological methods include preventive measures like exercise, external hip protectors, increase of dietary intake of calcium, vitamin $D$ and proteins, especially in elderly, over 65 years. Pharmacological measures are represented by different types of drugs, including biphosphonates, bone formation stimulatory drugs, agents with new mechanisms of action, hormone replacement therapy and they will be indicated only after a detailed clinical and paraclinical examination of the patient. Regardless of the chosen pharmacological measure, periodical follow-up of efficacy, side-effects and complications of antiosteoporotic treatment, by clinical examination and laboratory investigations targeting bone remodelling, is strongly indicated.

Keywords: osteoporosis, bone mineral density, fragility fractures

Received: 21 May 2013

Osteoporosis is the most frequent systemic bone disease, defined by the World Health Organization (WHO) in 1994 as a reduction of bone mass and microarchitectural deterioration of the bone tissue, resulting in an increased bone fragility and augmentation of fracture risk [1].

The main complication of osteoporosis is the bone fracture (vertebral and non-vertebral - hip, forearm etc.), the bone fracture risk during the lifetime of a postmenopausal woman reaching $30-40 \%$ [2].

From an etiopathogenic point of view osteoporosis may be classified into two categories $[3,4,5]$ :

- Primary osteoporosis caused by bone mass loss associated with age progression (in women and men) and menopause (in women). It can be divided into:

- type I, postmenopausal osteoporosis;

- type II, senile osteoporosis;

- juvenile osteoporosis, a rare form manifested during childhood.

- Secondary osteoporosis caused or exacerbated by diseases or drugs. Secondary causes appear especially in premenopausal women and in men, the prevalence in men being over $50-60 \%$.

Considering its large variety of causes and manifestations, the diagnostic and therapeutic approach of osteoporosis should be done only with the collaboration of

Correspondence to: Camelia Gliga

E-mail: m11gliga@yahoo.com physicians with different specialities, like family doctors, endocrinologists, gynecologists, rheumatologists etc.

Diagnosis of osteoporosis is based on symptomatology (in advanced stages complicated by fragility fractures), medical history, imagistic exploration and blood tests.

The symptomatology is dominated by diffuse pain or pain localised in the skeleton, mainly the spine, occuring most frequently in postmenopausal women (especially after the occurrence of fragility fractures or vertebral compaction) and more seldom in men.

A main element of symptomatology in osteoporotic patients is the presence of osteoporotic fracture, which is a pathologic fracture due to bone fragility occuring after minor efforts or traumas (sneezing, tilting, lifting of an object). Altough patients with osteoporosis may suffer fractures at any level of the skeleton, in some of its regions these fractures occur with predilection: in the distal part of the radius (Colles fracture), the vertebrae and femural neck.

In the modern medical practice for establishing the risk of osteoporosis and osteoporotic fracture, an easy-to-access online tool is increasingly used: FRAX (The Fracture Risk Assessment), which estimates the bone fracture risk at 10 years, taking into account a number of risk factors like gender, race, family medical history of osteoporosis, smoking, alcohol intake, diseases and treatments predisposing to osteoporosis $[6,7]$.

FRAX is a tool developed by the WHO. The purpose of FRAX is to offer the physician a simple and yet cheap med- 
ical evaluation method. Based on the calculated value, the physician will be able to assess whether the patient needs a treatment for osteoporosis.

But FRAX does not give any indications regarding the methods of treatment; it is an application that only suggests the need for further investigations (DEXA). It appreciates the probability of hip fracture risk and of major osteoporotic fractures (spine, forearm, hip or shoulder) for 10 years, only in persons older than 40 years. The FRAX has been developed after studying population cohorts from Europe, North America, Asia and Australia. From 2011 Romania has its own model of FRAX [6].

The National Osteoporotic Fundation (NOF) recommendations for treatment of osteoporosis considering DEXA and FRAX are as follows:

- patients with a history of fragility fracture (hip or vertebrae);

- lumbar or femural neck T-score $<-2.5$ determined using DEXA;

- osteopenia with high risk of fracture determined by FRAX (probability of hip fracture at 10 years $>3 \%$, probability of majore fracture at 10 years $>20 \%$ );

- a secondary cause of osteoporosis that increases the risk of fracture;

- history of any other fracture (except those of fragility) [2].

The most widely used technique of measuring bone mineral density, for an accurate diagnostic of osteoporosis is based on bone densitometry by DXA method (previously DEXA — dual energy X-ray absorptiometry) performed at the level of the lumbar spine and the hip. DXA measures the bone mineral density (BMD), the result being expressed by a T-score. This T-score shows the amount of bone a patient has, compared with that of a young adult of the same gender at his peak bone mass [8,9].

According to $\mathrm{WHO}$, depending on T-score, BMD will be appreciated as follows [10]:

- normal: T-score between +1 and -1 SD;

- osteopenia: T-score between -1 and $-2,5 \mathrm{SD}$;

- osteoporosis: T-score below or equal with -2,5 SD;

- severe osteoporosis: T-score below $-2,5$ and one or more fragility fractures.

Clinical applications of measuring bone densitometry by DXA are:

- diagnosis of osteoporosis;

- fracture risk assessment;

- treatment monitoring of osteoporosis.

Convetional X-ray is less sensitive in diagnosing osteoporosis without fractures but it is useful in diagnosing fragility fractures of the superior and inferior limbs and especially of the vertebrae. The compaction of vertebrae (wafer shape, fish shape, wedge shape) is a radiologcal sign of an osteoporotic fragility fracture.

The treatment of osteoporosis is complex involving preventive, non-pharmacological and pharmacological measures.

\section{Preventive non-pharmacological measures [11,12]:}

- Exercise performed regulary increases vertebral BMD, improves mobility, posture and prevents falls, thus assuring a better quality of life. Therefore, rounds of walking 3-5 times a week or jogging for 45-60 minutes are recommended, as well as home exercise programs to improve posture and strengthen the muscles (walking belt, stepper exercises), swimming, etc.

- Occupational therapy involves improving of daily physical activities and use of equipment at home (hallway handrails, non-slip solutions for stepping, shower chairs, etc.), necessary for prevention of falling downs.

- External hip protector worn by elder persons, was demonstrated to be useful in preventing age-related femural neck fractures.

- Falls prevention in persons over 65 years (by vitamin D supplementation, adequate treatment of cardiovascular diseases, impaired balance, impaired vision, avoiding sedatives etc.) will decrease the rate of osteoporotic fractures.

- Increase of dietary intake of calcium, vitamin D and proteins. Postmenopausal women and usually all persons over 65 years need a dietary supplementation of calcium and vitamin $\mathrm{D}$, in order to preserve the bone mass. The recommended daily calcium and vitamin $\mathrm{D}$ intakes in elderly people and postmenopausal women are as follows: $1200-1500 \mathrm{mg} /$ day of calcium and $400-800 \mathrm{mg} /$ day of vitamin D, doses that vary between different countries. Calcium and vitamin $\mathrm{D}$ intake is used in order to prevent osteoporosis but also to treat osteoporosis in associacion with the antiresorptive therapy [13].

The diet containing high levels of calcium includes dairy products, sardines, walnuts, sunflower seeds, tofu, kale, orange juice, etc. Excess of alcohol can interfere with calcium balance in the body by increasing the level of PTH and by inhibiting the enzyme that converts inactive vitamin $\mathrm{D}$ in its active form.

Endogenous production of vitamin $\mathrm{D}$ depends on the period of sun exposure time, on the normal functioning of the kidneys and liver. As a result, in elderly, an exposure to sunlight of 20 to 30 minutes per day, with uncovered head and arms and without the use of sunscreen against ultraviolet radiation, is enough to keep some good levels of vitamin D.

Nutritional protein deficiencies play a significant role in the development and worsening of osteoporosis in eldery and also in increasing of risk fracture. This is the reason for a protein rich diet in elderly, especially in those with manifest osteoporosis.

\section{Pharmacological measures}

Antiosteoporotic drug therapy is recommended, according to NOF protocols, in postmenopausal women and men 
over 50 years that present radiographically identified hip or vertebral fractures, femural neck or vertebral T-score of $-2,5$ or less, osteopenia with risk of fracture according to FRAX.

There are various treatment modalities:

- Antiresorptive therapy

- hormone replacement therapy (HRT)

- selective estrogen receptor modulators SERMs: raloxifen

- biphosphonates: alendronate, risendronate, ibandronate, zolendronate

- calcitonin

- Anabolic therapy (bone formation stimulators)

- parathyroid hormone (PTH)

- fluoride derivates (not in use anymore)

- anabolic steroids

- Therapy using agents with dual antiresorptive and anabolic action

- strontium ranelate

- Recent therapy, using agents with new mechanisms of action

- denosumab

\section{Hormon replacement therapy (HRT)}

Replacement therapy with estrogens or estrogen-progesterones was earlier the first choice therapy against osteoporosis in menopausal women $[8,14]$. Currently HRT is not recommended anymore as an antiosteoporotic treatment, but it is used in some patients to control postmenopausal symptoms.

\section{Therapy with selective estrogen receptor modulators \\ - SERMs}

The currently used SERM is raloxifene (Evista). SERMs mimic estrogens effect in some tissues (bones) and antiestrogens in others (endometrium, mammary gland).

Raloxifene decreseases bone turnover and prevents the loss of bone mass, thus decreasing the incidence of vertebral fractures with $35 \%$ [15]. It also reduces the prevalence of invasive breast cancer, lowers serum level of cholesterol and LDL cholesterol and does not produce endometrial bleeding and hyperplasia. The usual dose is of $60 \mathrm{mg} / \mathrm{day}$ orally. Raloxifene is indicated in young menopausal patients with no severe osteoporosis. As side effects heat waves and an increased incidence of profound venous thrombosis, were reported [15].

\section{Therapy with biphosphonates}

Biphosphonates are the most commonly used therapeutical agents in the treatment and prevention of osteoporosis. Biphosphonates have been widely used in the treatment of osteoporosis over the past 30 years. They are sinthetic analogues of pyrophosphate. Their main effect is the suppresion of bone resorption by osteoclasts and decreasing of bone turnover (which is increased in menopause).

Mechanism of action:

- binding to hydroxyapatite cristals at the level of active bone resorption surface, beneath the osteo- clasts, suppressing osteoclastic bone resorption

- reducing the activity of osteoclasts

- reducing activation of osteoclasts (suppresses recruiting and differentiation of precursors)

- acceleration of osteoclasts apoptosis.

Due to their bone tropism and resistance to enzymatic inactivation, biphosphonates are retained in the skeleton for a long time, thus allowing a prelonged time of action even after cessation of the treatment. Intestinal absorption of biphosphonates administered orally is low $(0,6 \%)$, towards intravenous administration with $100 \%$ absorption.

Side effects can be:

- digestive (esophagitis, esophageal ulcer, disphagia, abdominal pain)

- osteonecrosis of maxillary/mandibular bone occuring mainly in debilitated patients who undergo oral-maxilo-facial surgery

- atypical femure fractures (subtrochanterian) occuring after prolonged treatment with biphosphonates (approximately 5 years).

Contraindications are:

- esophageal anomalies (that delays esophageal transit)

- hipersensitivity to alendronates

- gastric ulcer

- uncorrected hypocalcaemia

- renal insufficiency.

Biphosphonates are represented by:

- Alendronate (Fosamax) administered in doses of 70 mg weekly, early in the morning before breakfast, with one glass of noncarbonated mineral water in orthostatic position. A combined formula of $70 \mathrm{mg}$ alendronate with $2800 \mathrm{IU}$ or $5600 \mathrm{IU}$ vitamin D (Fosavance) is also available. The drug is approved to treat osteoporosis in men, women at menopause and patients with glucocorticoid-induced osteoporosis. The efficacy of alendronate in reducing the risk fracture has been demonstrated both in the vertebrae and hips as well [10].

- Risendronate (Actonel, Risendros, etc.) administered in doses of $35 \mathrm{mg}$ once a week or $75 \mathrm{mg}$ on two consecutive days per week, under the same conditions like alendronate. Vertebral and hips antifracture efficacy has been demonstrated in case of this drug, too $[16,17]$.

- Ibandronate (Bonviva) is indicated in doses of 150 mg administered orally once a month or in intravenous injections in doses of $3 \mathrm{mg}$ every 3 months. The oral administration conditions of this drug are the same like in the other two already presented biphosphonates, antifracture efficacy being demonstrated especially in vertebral, but also in nonvertebral fractures $[14,16]$.

- Zolendronate (Aclasta) is a newer biphosphonate used in osteoporosis. It is indicated in doses of 5 $\mathrm{mg}$ once a year, in intravenous injections [8]. It is 
indicated in the treatment of osteoporosis in men, menopausal women and patients with glucocorticoid-induced osteoporosis. The anti-fracture effect was demonstrated both in vertebral and non-vertebral localizations. The reported side effect is an increased risk of acute renal failure requiring dialysis. Therefore the drug is contraindicated in patients with mild or severe renal failure (creatinin-clearence $<35 \mathrm{ml} / \mathrm{min}$ ) or in patients suffering from different renal diseases.

\section{Therapy with calcitonin}

Calcitonin is a polypeptid hormone that suppresses bone resorption and has analgesic properties. Currently used calcitonin products are those obtained from teleost fishes (eel, salmon), these being 50 to 100 times more potent than those obtained from other mammals. Antifracture efficacy has been demonstrated only in the verterbrae $[9,14]$. It represented a treatment option for those patients who can not use other available treatments against osteoporosis. In 2013, the EMA (European Medicines Agency) drew attention to the existence of an increased risk of malignancy associated with long-term administration of calcitonin compared to placebo. As a result the use of calcitonin in the treatment of postmenopausal osteoporosis was forbidden.

\section{Anabolic treatment (with parathyroid hormone, PTH)}

Secondary hyperparathyroidism resulting from decreased calcium absorption and subsequent increase in bone resorption is considered a major determinant of fractures in the elderly. However, a series of experiments demonstrated that PTH has also a strong anabolic action on the bones/ skeleton in case of its intermittent administration, in doses that lead to high serum concentrations (unlike with the continuous exposure to moderatly increased serum concentrations such as that occuring in hyperparathyroidism). Teriparatide is the compound that contains only the first part (amino acids 1-34) of the polypeptide structure of the parathyroid hormone (containing 84 amino acids). It exerts an intense anabolic effect on the bones and it is used in advanced formes of osteoporosis with high risk for fractures or in case of inefficient or intolerance to previous therapy. Lately, treatment with whole molecules of PTH $(1-84)$ is used. Because of the occurence of osteosarcoma in experiments on rats treated with very high doses of PTH, duration of treatment has been limited to 18 months, in the European Union, respective to 24 months in the USA. Both teriparatide and rhPTH 1-84 are administered as a daily subcutaneous injection $[2,17]$. Teriparatide is administered in doses of $20 \mathrm{mcg}$ per day, in subcutaneous injection. The duration of the treatment should not exceed 2 years.

\section{Therapy with strontium ranelate}

Mechanism of action of strontium ranelate involves a dual effect: mild reduction of bone resorption (by reducing the differentiation of pre-osteoclasts into osteoclasts) and maintenance or mild increase of bone formation (by increasing the replication of pre-osteoblasts into osteoblasts, leading to an increase in bone matrix synthesis). It is administered orally in form of the drug called Osseor, in doses of $2 \mathrm{~g}$ daily. It is indicated in treating osteoporosis in menopausal women with contraindications for biphosphonates (esophageal ulcers, erosions or stenosis, gastric ulcers), in women with unsatisfying response after more than one year of treatment with biphosphonates; more recently it was approved for treatment of osteoporosis in men. A contraindication of therapy with strontium ranelate is venous thromboembolism [18]. It is approved for treatment of osteoporosis in some European countries but it is not approved in the USA.

In 2013, the EMA, following the assessment of data demonstrating the association of this drug with an increased risk of heart disease, recommended certain restriction of its use only for the treatment of severe osteoporosis in postmenopausal women who are at increased risk of fracture or for the treatment of severe osteoporosis in men with increased risk of fracture. However in order to minimize the risk of the heart disease, they recommended additional measures including restricting its use in patients with cardiac or circulatory diseases.

\section{Therapy with new antiosteoporotic agents, with new} mechanisms of action (Denosumab)

Over the past decades, the discovery of receptor activator of nuclear factor - kappa B on the surface of pre-osteoclasts (RANK), of its ligand (RANKL) and of osteoprotegerin has been the key development in understanding osteoclast biology and the process of bone resorption. Denosumab is a human monoclonal antibody (IgG2) with high affinity for human RANKL, that blocks the binding of RANKL to RANK, thus blocking the differentiation of pre-osteoclasts in osteoclasts and also the activity of osteoclasts [19].

Denosumab (Prolia) is indicated for the treatment of osteoporosis in postmenopausal women with a medical history of osteoporotic fracture, with multiple risk factors for fracture, intolerance to other available antiosteoporotic therapies or in case of nonresponsive treatment with other antiosteoporotic drugs. It is used also in men with non-metastatic prostate cancer, using androgen deprivation therapy.

The follow-up of antiosteoporotic treatment efficacy will be periodically performed, every 3-6-12 months, by clinical examination (side effects, new fractures), laboratory investigations targeting bone remodelling (resorption and bone formation markers) and determining of bone mineral density by DXA, 1-2 years after antiosteoporotic therapy.

\section{References}

1. Kanis JA, McCloskey EV, Johansson $\mathrm{H}$, et al. A reference standard for the description of osteoporosis. Bone. 2008;42(3):467-75.

2. National Osteoporosis Foundation. Clinician's Guide to Prevention and Treatment of Osteoporosis. Available at http://www.nof.org/professionals/ clinical-guidelines. 2011 
3. Bono CM, Einhorn TA. Overview of osteoporosis: pathophysiology and determinants of bone strength. Eur Spine J. 2003;12(2):90-6.

4. Ringe JD, Farahmand P. Advances in the management of corticosteroidinduced osteoporosis with bisphosphonates. Clin Rheumatol. 2007;26(4):474-84.

5. Raisz LG. Pathogenesis of osteoporosis: concepts, conflicts, and prospects. J Clin Invest. 2005;115(12):3318-25.

6. http://www.shef.ac.uk/FRAX

7. Schwartz AV, Vittinghoff E, Bauer DC, et al. Association of BMD and FRAX score with risk of fracture in older adults with type 2 diabetes. JAMA. 2011;305(21):2184-92.

8. Lecart MP, Reginster JY. Current options for the management of postmenopausal osteoporosis. Expert Opin Pharmacother. 2011;12(16): 2533-52.

9. Sandhu SK, Hampson G. The pathogenesis, diagnosis, investigation and management of osteoporosis. J Clin Pathol. 2011;64(12):1042-50.

10. World Health Organization. WHO scientific group on the assessment of osteoporosis at primary health care level: summary meeting report. Available at http://www.who.int/chp/topics/Osteoporosis.pdf. 2012

11. Silverman SL. Selecting patients for osteoporosis therapy. Ann N Y Acad Sci. 2007;1117:264-72.
12. Warensjö E, Byberg L, Melhus $H$, et al. Dietary calcium intake and risk of fracture and osteoporosis: prospective longitudinal cohort study. BMJ. 2011;342: d1473.

13. Bischoff-Ferrari HA, Willett WC, Wong JB, et al. Prevention of nonvertebral fractures with oral vitamin $D$ and dose dependency: a meta-analysis of randomized controlled trials. Arch Intern Med. 2009;169(6):551-61.

14. Watts NB, Bilezikian JP, Camacho PM, et al. American Association of Clinical Endocrinologists Medical Guidelines for Clinical Practice for the diagnosis and treatment of postmenopausal osteoporosis. Endocr Pract. 2010;16(3):1-37.

15. Barrett-Connor E, Mosca L, Collins P, et al. Effects of raloxifene on cardiovascular events and breast cancer in postmenopausal women. N Engl J Med. 2006;355:125-37.

16. Geusens P. Bisphosphonates for postmenopausal osteoporosis: determining duration of treatment. Curr Osteoporos Rep 2009, 7 (1):12-7.

17. National Osteoporosis Foundation. Fast facts. Available at http://www. nof.org/node/40. 2012.

18. McClung MR, Lewiecki EM, Cohen SB, Bolognese MA, Woodson GC, Moffett $\mathrm{AH}$, et al. Denosumab in postmenopausal women with low bone mineral density. N Engl J Med. 2006;354(8):821-31.

19. Blake GM, Fogelman I. Long-term effect of strontium ranelate treatment on BMD. J Bone Miner Res. 2005;20(11):1901-4. 